\title{
Economic trade-off in the optimization of carrier aggregation with enhanced multi-band scheduling in LTE-Advanced scenarios
}

\author{
Daniel Robalo and Fernando J Velez
}

\begin{abstract}
This work proposes Long Term Evolution-Advanced (LTE-A) integrated Common Radio Resource Management (iCRRM) for inter-band carrier aggregation (CA) between band $7(2.6 \mathrm{GHz})$ and band $20(800 \mathrm{MHz})$, considering bandwidths of 5 and $20 \mathrm{MHz}$. The iCRRM entity performs component carrier (CC) scheduling and increases user's quality of service and experience while considering mobile video traffic. The performance from a new enhanced multi-band scheduling (EMBS) algorithm is compared to the one from a basic multi-band scheduler (BMBS), an integer programming-based general multi-band scheduling (GMBS) and the case without CA. EMBS involves reduced optimization scheduling complexity and allows the allocation of UEs to one or both CCs simultaneously, whereas both BMBS and GMBS only support one CC per UE. Simulations results have shown that, for $5 \mathrm{MHz} C \mathrm{Cs}$ and cell radius equal to 1,000 m, with EMBS and GMBS, the 3GPP and ITU-T's 1\% packet loss ratio (PLR) threshold is only exceeded above 58 UEs (goodputs of 7.48 and $7.40 \mathrm{Mbps}$, respectively), while with BMBS only 54 UEs (6.9 Mbps) are supported. Without CA, the minimum obtained PLR is approximately 2\%. For CCs with bandwidth of $20 \mathrm{MHz}$, only EMBS has been considered. The PLR threshold is not exceeded up to 40 users and the value of QoE raises from 2.86 (for 5-MHz bandwidth) to 3.96, while a gain of 9.56 occurs in supported goodput, increasing from 7.48 to $71.53 \mathrm{Mbps}$. Results from the cost/revenue trade-off have shown substantial improvements by using CA. Although the profit increases as the price per megabyte increases, it is verified that prices can be much lower if a bandwidth of $20 \mathrm{MHz}$ is available. Assuming values for the supported goodput under the PLR $\leq 1 \%$ range and $20 \mathrm{MHz} C \mathrm{Cs}$, it has been shown that the percentage of profit decreases at a considerably higher rate (compared to $5-\mathrm{MHz}$ bandwidth), due to the lower rate of decrease from the curve for costs. Considering PLR $\leq 1 \%$, the profit curve for $20 \mathrm{MHz} C \mathrm{Cs}$ at $0.001 € / \mathrm{MByte}$ is similar to the one for $5 \mathrm{MHz} C \mathrm{Cs}$ and price of $0.01 € /$ MByte for the smallest cell sizes (few hundreds of meters) but starts to decrease faster for larger cells.
\end{abstract}

Keywords: Carrier aggregation; Long Term Evolution-Advanced; Multi-band scheduler; Quality of service; Quality of experience; Radio resource management; Cost/revenue analysis

\section{Introduction}

To meet the increasing demand for wireless broadband services from fast-growing mobile users, aggregating frequency spectrum is one of the viable techniques to enhance data rates and improve service quality. The concept of spectrum aggregation is introduced by 3GPP in its LTE-Advanced (LTE-A), e.g. LTE R10. However, the introduction of spectrum aggregation or carrier aggregation (CA), as referred to in LTE R10, has required some

*Correspondence: fjv@ubi.pt

Instituto de Telecomunicações - Universidade da Beira Interior, Calçada Fonte do Lameiro, 6201-001 Covilhã, Portugal changes from the baseline LTE R8, although each component carrier (frequency band) in LTE-A remains backward compatible with LTE R8 as described in [1].

CA is considered as a key enabler for LTE-A [2], as it can meet or even exceed the IMT-Advanced requirement for large transmission bandwidth (40 to $100 \mathrm{MHz}$ ) and high peak data rate (500 Mbps in the uplink and 1 Gbps in the downlink) [3]. Each aggregated carrier is referred to as a component carrier (CC). The CCs can have bandwidths of $1.4,3,5,10,15$ or $20 \mathrm{MHz}$. A maximum of five CCs can be aggregated and can also be of different bandwidths. As such, the maximum aggregated

\section{Springer}

(C) 2015 Robalo and Velez. This is an Open Access article distributed under the terms of the Creative Commons Attribution License (http://creativecommons.org/licenses/by/4.0), which permits unrestricted use, distribution, and reproduction in any medium, provided the original work is properly credited. 
bandwidth is $100 \mathrm{MHz}$. User equipment (UE) may simultaneously receive or transmit data on one or multiple CCs, whereas in the 3GPP R8 specifications [4], each UE uses only one $\mathrm{CC}$ to communicate at one time. Moreover, since it is important to keep backward compatibility with R8 and R9 UEs, the aggregation is based on R8/R9 carriers and can be used for both FDD and TDD modes.

The easiest way to arrange aggregation would be to use contiguous component carriers within the same operating frequency band (as defined for LTE), the so called intra-band contiguous. However, in practice, such a large portion of continuous spectrum is rarely available. CA, where multiple CCs of smaller bandwidth are aggregated, is an attractive alternative to increase data rate. Additional advantages are offered by CA in terms of spectrum efficiency, deployment flexibility, backward compatibility and more. By aggregating non-contiguous carriers, fragmented spectrum can be more efficiently utilized [5]. For non-contiguous allocation, it could either be intraband, i.e. the CCs belong to the same operating frequency band, but have a gap, or gaps, in between, or it could be inter-band, in which case the CCs belong to different operating frequency bands [6]. In $[7,8]$ and [9], the authors addressed the problem of how to optimize the resource allocation process in a multi-carrier system, while maintaining low complexity. Both simple theoretical and simulation results were obtained, which show that with low number of users and low percentage of LTE-A users, the load balancing method of round robin (RR) achieves better performance than the mobile hashing $(\mathrm{MH})$ balancing. It was also found that using independent packet scheduling per CC suffers from poor coverage performance. In this context, the authors proposed a cross CC packet scheduler algorithm, which is a simple extension of the existing proportional fair scheduler. The cross CC algorithm is aware of the user throughput over all the aggregated CCs. As a result, it was shown that the cross $\mathrm{CC}$ algorithm maximizes the network utility even if users are provided with different number of CCs. This approach however only accounts for the cell throughput and disregards QoS parameters, e.g. delay and loss. Besides, full buffer traffic is addressed, which is not representative of nowadays and future cellular network traffic. In [10], a scheduling strategy for CA using pre-organized resource block (RB) sets was presented. An analytical evaluation framework was performed to determine the expected number of RBs required by users, based on a mapping of Channel Quality Indicator (CQI) values to data rates per $\mathrm{RB}$ and the statistical behaviour of the CQI. RBs can be grouped into sets based on the predefined maximum number of RBs and spectrum availability. By scheduling these RB sets, this scheme can help to reduce the scheduling delay. Nevertheless, this adds further scheduling complexity due to the RB pre-organization functionality. In this context, this work addresses LTE-A CA and proposes an updated integrated Common Radio Resource Management (iCRRM), from [11], that performs CC scheduling to satisfy the user's quality of service (QoS) and experience (QoE) requirements while maximizing spectral efficiency. Two inter-band $\mathrm{CA}$, band $7(2.6 \mathrm{GHz})$ and band $20(800 \mathrm{MHz})$, are considered. As stated above, large portions of continuous spectrum are rarely available and the aggregation of smaller bandwidths is an attractive solution to reduce spectrum under utilization. In 2011, the Portuguese telecommunications regulator, ANACOM, auctioned LTE bands 7 and 20. As a consequence, in Portugal, only $5 \mathrm{MHz}$ ("lots") can be made available for the considered CCs [12]. In South Korea, LG Uplus Corp, the third mobile carrier, created the LTE-A service by combining bandwidth of $40 \mathrm{MHz}$ at $2.6 \mathrm{GHz}$, plus $20-\mathrm{MHz}$ bandwidths in the $800-\mathrm{MHz}$ and $2.1-\mathrm{GHz}$ bands [13]. Also in UK, Hong Kong and Singapore, late 2013 or in early 2014, EE, CSL or Sing Tel Mobile operators launched LTE-A carrier aggregation services, bringing together $20 \mathrm{MHz}$ from $1,800-\mathrm{MHz}$ spectrum band and $20 \mathrm{MHz}$ from 2.6-GHz spectrum band [14-16]. Since November 2014, Telekom Deutschland has been offering higher date rates by using the $2,600-\mathrm{MHz}$ frequency band in combination with the $800-\mathrm{MHz}$ band, with 20$\mathrm{MHz}$ bandwidths [17]. Also, 5-MHz and 20-MHz bandwidth CCs are considered for this research while assuming inter-band carrier aggregation between the $800-\mathrm{MHz}$ and 2.6-GHz frequency bands. Besides, following the forecast from [18], in this work, two different types of video traffic are addressed under the premise that in 2013 it represented more than half (53\%) of the total traffic and will reach $69 \%$ of all worldwide mobile data traffic by 2018 .

Furthermore, a normalized transmitter power formulation is considered. On the one hand, this formulation allows for computing the required eNB power that enables to maintain a constant average cell SINR for different cell radii, hence to obtain comparable CA results for different cell radii. On the other hand, the formulation guarantees lower energy consumptions, e.g. small cell radii require lower transmitter power than their counterpart with larger radii, to achieve comparable values for SINR and coverage.

By considering extensive simulation results, the iCRRM performance metrics, i.e. packet loss, delay, goodput and user's expected quality of experience, are analysed and compared with a CRRM, which performs basic multiband scheduling, and the summed capacity of two CC LTE systems, i.e. without CA. Finally, the optimization of economic trade-off is pivotal in the optimization of $4 \mathrm{G}$ mobile communication systems. The cost/revenue analysis shows the performance and economic gain achieved with all multi-band schedulers while comparing their performance with the one from the system without CA. 


\section{Common radio resource management for carrier aggregation}

\section{Objective and system model}

The RRM framework for LTE-A retains many similarities with the ones from LTE. With CA, however, in every transmission time interval (TTI), it becomes possible to simultaneously schedule a user on multiple CCs, each of which may exhibit different radio channel characteristics. Supporting multi-CC operations introduces therefore some new challenging issues in RRM framework for LTE-A systems [19].

Figure 1 illustrates the RRM structure for a multicomponent carrier LTE-A system. The eNB first performs admission control to decide which users to serve and then employs layer-3 $\mathrm{CC}$ selection to allocate the users on different CCs [7]. Once the users are assigned onto certain $\mathrm{CC}(\mathrm{s})$, the layer-2 packet scheduling (PS) is performed. In order to allow for backward compatibility so LTE and LTE-A users can co-exist, it has been decided to use independent layer-1 transmissions, which contain link adaptation (LA) and hybrid automatic repeat request (HARQ) etc, per CC, in line with the LTE assumptions mentioned in [1].

The use of an independent link adaptation for each $\mathrm{CC}$ may help to optimize transmission according to radio conditions. Different levels of coverage can be provided by setting each $\mathrm{CC}$ with its own transmission power. This is especially the case from inter-band CA, since the radio channel characteristics, such as propagation, path loss and building penetration loss, may vary according to the operating radio frequency bands, i.e. selecting different transmission parameters, such as modulation scheme, code rate and transmit power per CC, it is expected to be useful to further improve user QoS. When the user receives packets, it estimates the SINR for the received signal, estimates channel quality and converts it a set of CQI feedbacks reported to the eNB for each $\mathrm{CC}$, following the conventions shown in Figure 1. During the simulations, each eNB maintains the list of UEs associated to it, storing, for each of them, the ID and the latest CQI feedbacks.

In the context of this research, one investigates noncontiguous inter-band CA from an upper layer point of view and proposes an integrated CRRM (iCRRM) entity where CRRM and CA functionalities are performed together. First, by using an integer programming (IP)-based algorithm, the inter-frequency handovers are achieved in an optimized way, performing the scheduling via the optimal solution of a general multi-band scheduling (GMBS) problem. Additionally, due to the complexity and the impossibility of allocating UEs to more than one CC, in the GMBS algorithm proposed in [11], an enhanced multi-band scheduling (EMBS) algorithm is alternatively incorporated into the iCRRM entity. This EMBS performs the scheduling functionalities instead of the GMBS with significantly reduced complexity while facilitating to allocate UEs to multiple CCs.

The employed resource allocation (RA) algorithms allocate the user packets to the available radio resources in order to satisfy the user requirements and to ensure efficient packet transport, e.g. minimize loss or maximize spectral efficiency. The RA is envisioned to have an inherent tuning flexibility to maximize the spectral efficiency of the system for any type of traffic QoS and QoE requirements. The RA adopted here maps packets of variable size into CCs for transmission over the physical (PHY) layer depending on the channel quality.

The proposed iCRRM enables the integration of spectrum and network resource management functionalities that leads to higher performance and system capacity gains and is the novelty from the proposed approach. The key to such integration is the pooling of resources together; the integration allows for mapping of service requirements onto an available spectrum amount and translates the latter into network load. As stated above, the iCRRM uses inter-band aggregation to achieve shorter delay and higher user throughput, by exploiting the

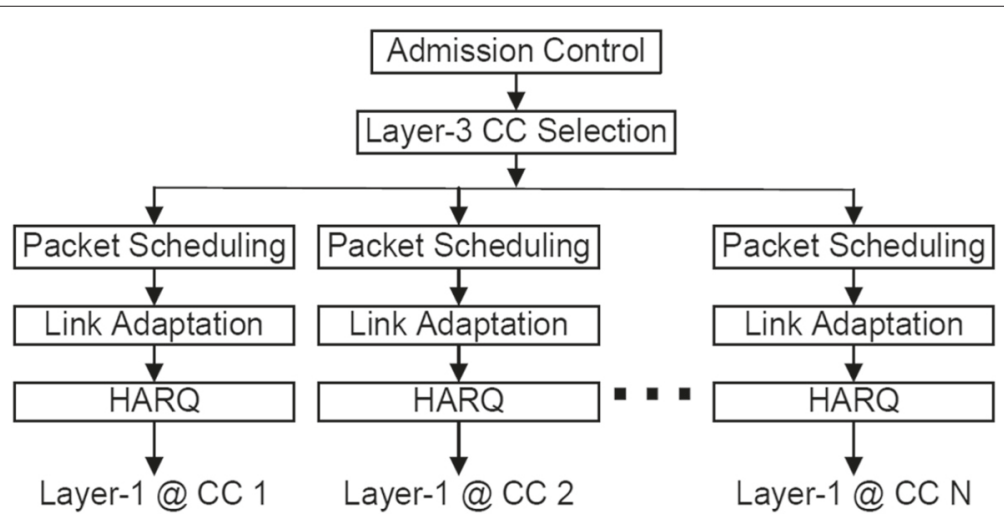

Figure 1 Structure of a multi-component carrier LTE-A system (extracted from [7]). 
channel diversity. These bands show independent Channel Quality Indicators (CQIs) over time and space, which becomes a source of diversity at the PHY layer, with an important chance to achieve higher spectrum efficiency. Information from the network about the system state (e.g. received signal strength, transmitted power, UEs velocity etc.) are used in RRM as well as in procedures such as load, admission and congestion control, which can successfully be combined with dynamic spectrum use.

In the context of CA in an LTE-A scenario, two frequency bands, i.e. two CCs, are available to the operator. The network is deployed with two collocated omnidirectional hexagonal coverage zones with radio frequency band 7 and 20, i.e. $2.6 \mathrm{GHz}$ and $800 \mathrm{MHz}$. The addressed scenario resembles the 3GPP scenario 2, from [4]. The corresponding first tier of interferers has been considered. Although propagation loss is higher at $2.6 \mathrm{GHz}$, through transmitter power tuning [20], a constant average signal-to-interference-plus-noise ratio (SINR) is guaranteed in this study. Thus, comparable results between CCs are assured. For future reference, it is worthwhile to note that a full radio access network (RAN) infrastructure sharing configuration [21] is assumed, e.g. the mast, eNB and Mobility Management Entity (MME/GW) are shared by both CCs. The addressed scenario and infrastructure sharing configuration are shown in Figure 2, an example for reuse pattern three.

The radio channel considered follows the ITU radio propagation COST-231 Hata model, for macro cell propagation scenarios in urban and suburban areas, outside the high rise core where the buildings are of nearly uniform height [22]. The channel loss between the UE and the eNB is modelled by using a shadowing loss with lognormal distribution and by considering fast fading with Jakes model. The COST-231 Hata path loss (PL) model is given by:

$$
\begin{aligned}
L_{[\mathrm{dB}]}=40 \cdot\left(1-4 \cdot 10^{-3} \cdot D_{\mathrm{hb}[\mathrm{m}]}\right) \log _{10}\left(R_{[\mathrm{km}]}\right) & -18 \cdot \log _{10}\left(D_{\mathrm{hb}[\mathrm{m})}\right)+ \\
& +21 \cdot \log _{10}\left(f_{[\mathrm{MHz}]}\right)+80(1)
\end{aligned}
$$

where $R$ is the eNB - UE separation in kilometres, $f$ is the carrier frequency and $D_{h b}=15 \mathrm{~m}$ is the base station antenna height, measured from the average rooftop level. The interference in the UE is calculated by considering the signal strength received from the first ring of neighbouring BSs and the thermal noise. The parameters and models assumed for both the $800 \mathrm{MHz}$ and $2.6 \mathrm{GHz} \mathrm{CCs}$ are shown in Table 1.

\section{Downlink packet scheduler}

One of the LTE simulator [23] that implemented downlink packet schedulers computes metrics for the allocated $\mathrm{CC} /$ band RBs and assigns them according to the highest metric value. Well-known downlink packet scheduling strategies, such as proportional fair, modified largest weighted delay first (M-LWDF) and exponential proportional fair, have also been implemented into LTE-Sim. The study of these opportunistic packet scheduling algorithms is beyond the scope of this paper. However, through extensive simulations, it has been found that in the context of addressed video applications, the M-LWDF scheduler outperforms the others [23] and will be considered for the purpose of capacity analysis in this research. As discussed in [23], M-LWDF is an algorithm designed to support multiple real-time data users and supports multiple data users with different QoS requirements. In every transmission time interval (TTI), the scheduler computes a metric, $w_{i, j}$, for the $i$ th flow in the $j$ th sub-channel. If the $i$ th flow is a real-time flow, the metric is computed as follows [23]:

$$
w_{i, j}=a_{i} D_{\mathrm{HOL}, i} \times \frac{r_{i, j}}{\bar{R}_{i}}
$$

where $D_{\mathrm{HOL}, i}$ is the $i$ th flow head of line (HOL) packet delay, $r_{i, j}$ is the instantaneous available rate (of the $i$ th



Figure 2 Inter-band carrier aggregation infrastructure sharing configuration and deployment scenario with two collocated hexagonal coverage zones using different frequency bands (example for reuse pattern three). 
Table 1 Parameters and models used for the $800 \mathrm{MHz}$ and $2.6 \mathrm{GHz}$ bands

\begin{tabular}{|c|c|c|}
\hline Carrier frequency & $800 \mathrm{MHz}$ (band 7) & $2.6 \mathrm{GHz}$ (band 20) \\
\hline Bandwidth $[\mathrm{MHz}]$ & 5 or 20 & 5 or 20 \\
\hline Path loss model & $L_{800 \mathrm{MHz}}=119.8+37.6 \log _{10}(R)$ & $L_{2.6 \mathrm{GHz}}=130.5+37.6 \log _{10}(R)$ \\
\hline Omni. antenna gain [dBi] & 14 & 14 \\
\hline
\end{tabular}

flow in the $j$ th sub-channel) and $\bar{R}_{i}$ is $i$ th flow average transmission rate, computed as follows [23]:

$$
\bar{R}_{i}=0.8 \bar{R}_{i}(k-1)+0.2 R_{i}(k)
$$

where $R_{i}(k)$ is the data rate achieved by the $i$ th flow during the $k$ th TTI and $\bar{R}_{i}(k-1)$ is the data rate estimation in the previous TTI.

Given two flows with equal HOL, $\alpha_{i}$ weights the metric so that the user with the strongest requirements in terms of acceptable loss rate and deadline expiration will be preferred for allocation [24] and is given by [23]:

$$
\alpha_{i}=-\frac{\log \left(\delta_{i}\right)}{\tau_{i}}
$$

where $\tau_{i}$ is packet delay threshold and $\delta_{i}$ is maximum probability that $D_{\mathrm{HOL}, i}$ exceeds the delay threshold of the $i$ th flow, respectively.

Although it will not be the case in this research, it is worthwhile to mention that, for non-real-time flows, the metric is computed as are the ones from the proportional fair scheduler, mentioned in [23].

\section{Multi-band scheduling}

Scheduling decisions are strictly related to the channel quality. With the extensions addressed in the physical layer, UEs are able to periodically measure the experienced channel quality of both CCs, create CQI feedbacks and transmit them to the eNB during the UL. These feedbacks on each CC is of fundamental importance for proper allocation and of distribution of resources from LTE-Sim [23] among users. Hence, the downlink packet scheduler class has also been extended to implement three multi-band scheduling in the context of CA.

Three downlink multi-band schedulers have been implemented so far. The general multi-band scheduling (GMBS) problem (proposed in [20]) is solved with integer programming (IP), using binary variables, as it is formulated by a general assignment problem (GAP) [25]. On the one hand, GMBS aims at maximizing the cell goodput, considering the video service bit rate, bit error rate and achievable DL throughput according to the supported Modulation and Coding Scheme (MCS). On the other, GMBS only allows to allocate UEs to one of the available CC at a time, i.e. TTI. Further details on GMBS are available in [20].
For comparison purposes, a basic multi-band scheduling (BMBS) that implements basic common RRM functionalities has also been included in LTE-Sim. BMBS aims allocating UEs to a preselected/primary CC, say band 20 $(800 \mathrm{MHz})$, until the maximum load that can be handled in the band, $L_{b}^{\max }$ is reached. Beyond this capacity threshold, the remaining UEs are allocated to the second CC, say band 7 (2.6 GHz). Similarly to GMBS, UEs can only be allocated to one band at each TTI. The performance and average cell capacity (packet loss ratio, delay and goodput) of both GMBS (integer programming approach) and BMBS have been addressed in [20] within the framework of simulations performed with LTE-Sim.

One of the main issues of the above-mentioned multiband scheduler is the increased complexity of the optimization process, specifically when the number of UEs in the network growths. Besides, this scheduler does not consider the possibility of UEs to use more than one CC at the same time, unlike 3GPP specifications for LTE-A, LTE R9 and above. Although it is arguable that the profit function (PF) could be modified to consider allocating UEs to multiple CCs, this would imply that an allocation variable $x_{b, u}$ should be computed for all RBs instead of (in this case) two CCs, e.g. considering two $5 \mathrm{MHz} \mathrm{CCs}$ $(25 \mathrm{RBs} \times 2)$, the PF should compute 50 allocation variables per UE. Therefore, the computation complexity of resource scheduling (optimization) would become unacceptable. Besides, both of these downsides have already been identified in the literature, in [1]. Hence, to overcome the limitations of the previous schedulers, the enhanced multi-band scheduling (EMBS) has been developed. On the one hand, IP optimization is no longer employed, instead a more traditional scheduling approach is used, i.e. a scheduling metric for each $\mathrm{RB}$ of each $\mathrm{CC}$ is computed. In return, the RB allocation is performed according to the highest value obtained. On the other hand, this approach allows allocating UE in either or both bands simultaneously, e.g. according to the metric value.

The scheduling metric is computed as follows:

$$
w_{i, j, b}=D_{\mathrm{HOL}, i} \times \frac{R\left(\mathrm{CQI}_{i, j, b}\right)^{2}}{\bar{R}_{i} \times S_{\text {rate }}}
$$

where $D_{\mathrm{HOL}, i}$ and $\bar{R}_{i}$ stand for the same, as above (for the M-LWDF), $S_{\text {rate }}$ is the video bit rate and $R\left(C Q I_{i, j, b}\right)$ is the DL throughput of band/CC $b$ for the $i$ th flow in the $j$ th sub-channel as a function of the supported MCS. 
Hence, the channel diversity of both CCs is also accounted for during the scheduling (RB allocation) process. In this case, $D_{\mathrm{HOL}, i}$ insures that video flows/UEs with the higher delay, i.e. the difference between the time in which the transmission was requested and the current simulation time, obtain a higher metric value. $R\left(\mathrm{CQI}_{i, j, b}\right)$ is squared to guarantee that RBs with higher CQIs achieve a higher metric value, and as a consequence higher data rates should be obtained.

\section{Simulation environment}

In this work, the CA gain is evaluated for several inter-cell distances with frequency reuse pattern three. In order to have comparable results and to reduce energy consumption, by reducing the transmitter power for low cell radii, CA is analysed at constant average cell SINR. The method to compute the normalized transmitter power required to maintain such average SINR, i.e. the transmitted power for each cell radii so they all have a similar average cell SINR, was described in our previous works [11] and [20] and considered within the simulation framework of this research.

To study the performance of the proposed iCRRM, several LTE system level simulations have been performed within a LTE-A scenario. The comparison parameters include the average cell supported goodput, delay, packet loss ratio (PLR) and spectral efficiency. The LTE simulator chosen to perform this evaluation is LTE-Sim [23], developed by the University of Bari. LTE-Sim is an event-driven simulator, written in $\mathrm{C}++$, using the objectoriented paradigm. Several traffic generators at the application layer have been implemented, and the management of data radio bearer is supported. In particular, the video traffic addressed in this research is a trace-based application which sends packets based on realistic video trace files. To study the performance of the proposed iCRRM, the inter-band CA scenario shown in Figure 2 is considered. UEs are constantly moving at $3 \mathrm{kmph}$ using LTE-Sim random direction mobility model. Each UE only uses one video flow, the H.264 128-kbps video bit rate flow (as described in [23]) when 5-MHz bandwidth is assumed for the CCs and H.264 3.1-Mbps video clip if 20-MHz bandwidth is considered. Maximum delay of $1 \mathrm{~ms}$ is considered. LTE-Sim [23] provides a support for radio resource allocation in a time-frequency domain and, in this configuration, the duration of one LTE radio frame is $10 \mathrm{~ms}$. One frame is divided into ten sub-frames of $1 \mathrm{~ms}$ each, and each sub-frame is divided into two slots of $0.5 \mathrm{~ms}$ each. Each slot contains either six or seven OFDM symbols, depending on the cyclic prefix (CP) length [26]. The normal $\mathrm{CP}$ is used in urban cells and high data rate applications while the extended $\mathrm{CP}$ is used in special cases like multi-cell broadcast and in very large cells (e.g. rural areas, low data rate applications).
Furthermore, LTE radio resources are allocated in units of RBs or physical RBs (PRBs). Each PRB contains 12 subcarriers and one slot. If the normal CP is used, a PRB will contain 12 subcarriers over seven symbols. If the extended $\mathrm{CP}$ is used, the PRB contains only six symbols. In the context of CA, normal CP frames is assumed, each carrier band has a $5-\mathrm{MHz}$ bandwidth and thus a total of $25+25=$ 50 PRBs are available for scheduling. An overview of the simulation parameters is presented in Table 2.

\section{Simulation results}

The evaluation of the proposed iCRRM entity involved evaluating the performance of several simulation scenarios:

1. Two LTE systems operating separately at $800 \mathrm{MHz}$ and $2.6 \mathrm{GHz}$, i.e. without $\mathrm{CA}$, considering the $\mathrm{M}-\mathrm{LWDF}$ scheduler and either $5-\mathrm{MHz}$ or $20-\mathrm{MHz}$ bandwidths;

2. One LTE-A scenario with both frequency bands managed with basic CRRM functionalities (basic multi-band scheduling), only considering a bandwidth of $5 \mathrm{MHz}$

3. One LTE-A scenario with both frequency bands managed with the proposed iCRRM entity:

(a) One set performed with the general multi-band scheduler, only considering a bandwidth of $5 \mathrm{MHz}$;

(b) One set performed with the enhanced multi-band scheduler, bandwidths of either $5 \mathrm{MHz}$ or $20 \mathrm{MHz}$.

Each scenario was simulated 20 times, these results have been averaged and the confidence intervals have been determined. In the case of $5-\mathrm{MHz}$ bandwidth, the analysis of average cell PLR and delay are performed by averaging the results from scenario 1 while comparing them with

\section{Table 2 Simulation parameters}

\begin{tabular}{ll}
\hline Simulation parameters & \\
\hline Reuse pattern & 3 \\
Simulation duration & $46 \mathrm{~s}$ \\
Flow duration & $40 \mathrm{~s}$ \\
Frame structure & $\mathrm{FDD}$ \\
Bandwidth & $5 \mathrm{MHz}$ or $20 \mathrm{MHz}$ per CC \\
Slot duration & $0.5 \mathrm{~ms}$ \\
Scheduling time (TTI) & $1 \mathrm{~ms}$ \\
Number of RBs & $25 \mathrm{RB}$ per CC \\
Maximum delay & $150 \mathrm{~ms}$ \\
Video bitrate & $128 \mathrm{kbps}$ or $3.1 \mathrm{Mbps}$ \\
UE mobility & random direction, $3 \mathrm{kmph}$ \\
\hline
\end{tabular}


the ones from scenarios 2 and 3. In terms of average cellsupported goodput analyses, the system capacity obtained in scenario 1 is summed and compared with the results from scenarios 2 and 3. Furthermore, it is worthwhile to note that the following results obtained without CA have been compared and are well within the range of ones obtained in the packet scheduling algorithms studies performed in [23,26] and [24]. Innovative results considering a bandwidth of $20 \mathrm{MHz}$ are compared between scenario 1, without CA, and scenario 3(a), which only considers the EMBS proposal.

\section{Packet loss ratio}

Figure 3 shows the average cell PLR as a function of the number of UEs for a cell with $R=1,000 \mathrm{~m}$. A bandwidth of $5 \mathrm{MHz}$ and the H.264 128-kbps video flow have been initially considered. As expected, both CRRM (BMBS) and iCRRM outperform the scenario without CA.

However, according to the ITU-T G.1010 [27] and 3GPP TS 22.105 [28] recommendations, the PLR should not exceed $1 \%$. In this context, the $1 \%$ PLR threshold is only exceeded above approximately 58 UEs with iCRRM general (GMBS) and enhanced (EMBS) multi-band scheduler ( $1.03 \%$ and $0.88 \%$, respectively), whereas CRRM only supports up to 54 UEs (0.99\%). Without CA, the minimum obtained PLR is approximately $2 \%$. Besides, it is also verified that overall, the EMBS enables to obtain values for the PLR lower than the ones with the GMBS. Figure 4 shows again the obtained results by magnifying Figure 3 . The average PLR without CA (Average $2.6 \mathrm{GHz}$ and $800 \mathrm{MHz}$ ) is not shown, since the minimum obtained value is superior to $2 \%$, which is the maximum value shown for PLR.

Additionally, the 20-MHz bandwidth scenario has been assumed together with the use of the H.264 3.1-Mbps video flow. Figure 5 shows the average cell PLR as a function of the number of UEs for cell radius $R=1,000 \mathrm{~m}$. In this case, the 1\% PLR threshold is only exceeded above approximately $40 \mathrm{UEs}(\mathrm{PLR}=0.65 \%)$. This clearly shows that, with EMBS, PLR considerably decreases.

\section{Delay}

The average cell delay for $R=1,000 \mathrm{~m}$ is shown in Figure 6 for the scenario with $5-\mathrm{MHz}$ bandwidth. As in the previous case, both CRRM (BMBS) and iCRRM present better results than without $\mathrm{CA}$, i.e. lower delay. Moreover, iCRRM's EMBS outperforms the GMBS. Similarly to the PLR, ITU-T G.1010 [27] and 3GPP TS 22.105 [28] also define delay performance targets, i.e. 150 -ms preferred and 400-ms limit delay. For the considered number of UE, neither of these targets is exceeded. Nonetheless, when the previous $1 \%$ performance target is exceeded, i.e. 54 and 58 UEs, with CRRM and iCRRM (EMBS and GMBS), respectively, the achieved delay for the $5-\mathrm{MHz}$ bandwidth case is approximately 11.22, 11.44 and $7.68 \mathrm{~ms}$ with CRRM and iCRRM's GMBS and EMBS scheduler, respectively. Without $\mathrm{CA}$, the average cell delay is always superior to the ones from the above cases.

It is worthwhile to compare the results from the $5-\mathrm{MHz}$ bandwidth scenario with the ones from the 20-MHz bandwidth one. Figure 7 shows the average cell delay for $R=$ $1,000 \mathrm{~m}$ while comparing the cases without CA and the use of EMBS for both values of the bandwidth.

With EMBS, when the 1\% PLR performance target is exceeded, i.e. 54 and 40 UEs using $5 \mathrm{MHz}$ and $20 \mathrm{MHz}$ CCs, respectively, the achieved delay is approximately 8 and $2.4 \mathrm{~ms}$ (128 kbps and 3.1 Mbps video clips, respectively). Without CA, the average cell delay is always considerably superior to the ones from the previous cases.



Figure 3 Comparison of the average cell PLR as function of the number of UEs for $R=1,000 \mathrm{~m}$ (by applying CRRM or iCRRM the PLR substantially decreases, compared to the case without CA). 


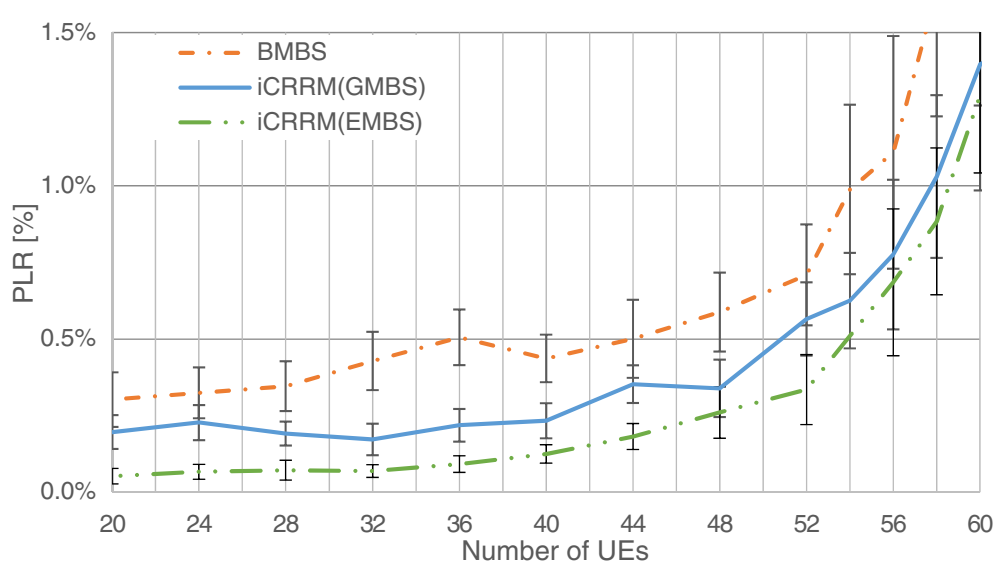

Figure 4 Magnified refitting of the average cell PLR as function of the number of UEs, where only the range of interest is shown for PLR and number of UES.

\section{Quality of experience}

The permanent evolution of wireless network technologies allows for improved data rates and coverage areas while facilitating new multimedia and mobile services. Considering this evolution of services and applications, operator's success does not only depend on their QoS but also if it meets the end user's expectations. With the increasing competition, improving the quality of the offered services, as perceived by the users (QoE), becomes important as well as a significant challenge to service providers with a goal to minimize the customer churn yet maintaining their competitive edge [29]. However, QoS is generally defined in terms of network delivery capacity and resource availability but not in terms of the satisfaction to the end-user. QoE is very subjective in nature, the most accurate approach to evaluate it is the subjective quality assessment, since there is no better indicator of personal quality than the one given by a human being.
The existing works cited in $[8,19,30,31]$ mainly focus on the quality of service (QoS) performance of CA system. However, these days, service providers are switching the focus from network QoS to user quality of experience (QoE) to provide their services in the most cost- and resource-efficient manner with ensured user satisfaction. Therefore, the resource allocation algorithm based on various QoE contributing factors such as throughput, jitter, cost and reliability should be required in the process of resource allocation for the CA system.

It is important that QoE is expressed as a function of the network and equipment that influence user behaviour and result in a certain level of QoE. Therefore, QoE data should succeed whenever possible in combining both user experience and technical measures; for example, to provide an equation for the user experience when using a particular service with known levels of QoS [32]. As such, QoS metrics gathered from various parts of the network



Figure 5 Comparison of the average cell PLR, as a function of the number of UEs, for CA between scenarios with 5 and 20 MHz CCs (the case with the separate use of two bands, i.e. without CA, is considered as a reference). 


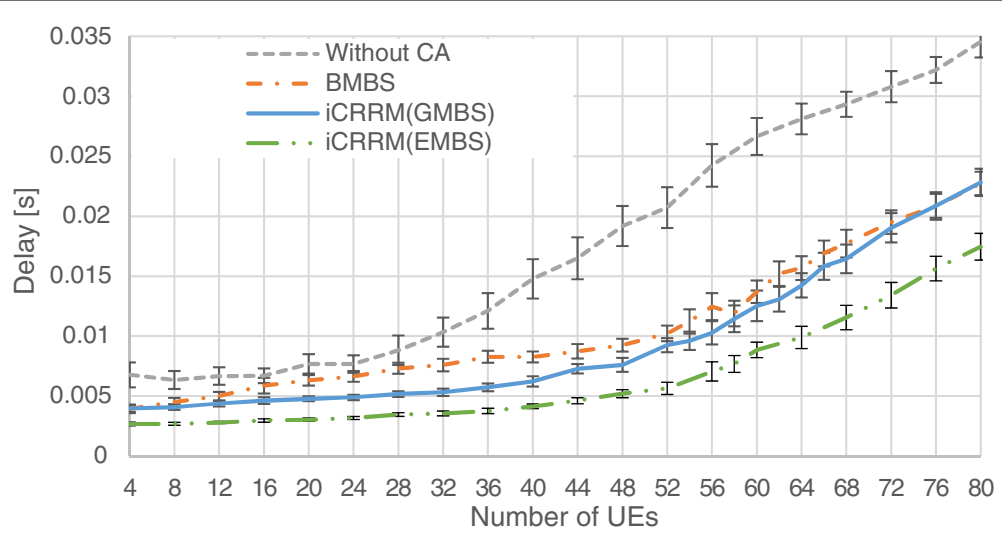

Figure 6 Average cell delay in a cell as a function of the number of UEs for $R=1,000 \mathrm{~m}$ (by applying CRRM or iCRRM, the delay decreases, compared to the case without CA).

must be mapped onto QoE targets, facilitating the inclusion of the end-user perception into the QoE model. In this context, besides assessing the network service level parameters, the QoE can also be evaluated by employing the model for the mapping between QoS and QoE proposed in [33]. This model addresses multimedia applications, gaming, video, web-browsing and audio. The video sub-model is based on the mean opinion score (MOS) measurements which are mathematically fitted to obtain the following equation:

$$
\begin{aligned}
\mathrm{QoE}= & 3.2147-0.00266916 \times b_{\text {rate }}-10.4811 \\
& \times d-20.9894 \times \rho-5.8875 \times 10^{-6} \\
& \times b_{\text {rate }}^{2}+40.3305 \times d^{2}+166.121 \times \rho^{2}+1.449 \\
& \times 10^{-8} \times b_{\text {rate }}^{3}-42.493 \times d^{3}-730.016 \\
& \times \rho^{-3}-4.2939 \times 10^{-12} \times b_{\text {rate }}^{4}+18.3884 \times d^{4} \\
& +1764.47 \times \rho^{4}-2.29851 \times 10^{-15} \times b_{\text {rate }}^{5} \\
& -3.48213 \times d^{5}-2069.09 \times \rho^{5}+8.08679 \times 10^{-19} \\
& \times b_{\text {rate }}^{6}+0.237418 \times d^{6}+903.102 \times \rho^{6}
\end{aligned}
$$

where $d$ is the delay, in ms, $\rho$ is the percentage of loss and $b_{\text {rate }}$ is the video bitrate, in kilobit per second. The goodness of fit is confirmed by the coefficient of determination, $R^{2}=0.84$, and the mean square error (MSE), MSE $=0.197$.

Considering this model and previous average cell PLR and delay simulation results (with $b_{\text {rate }}=128 \mathrm{kbps}$ ), Figure 8 shows the predicted average cell QoE as a function of the number of active UEs in cells with $R=1,000 \mathrm{~m}$.

From Figure 8, it is clear that employing CA improves the average cell QoE. Without CA, an average QoE of 2.7 is obtained below 28 UEs; beyond this values, the quality substantially decreases and reaches its lower value with the maximum considered UEs. With CA, as expected by the obtained PLR and delay, the EMBS provides the better results followed by the GMBS and CRRM (BMBS). Moreover, it is interesting to note that, as expected by ITU-T G.1010 [27] and 3GPP TS 22.105 [28], the higher decline of the estimated QoE value occurs approximately with the same number of UEs from which the 1\% PLR is exceeded, i.e. 54 and 58 UEs with CRRM and iCRRM, respectively,

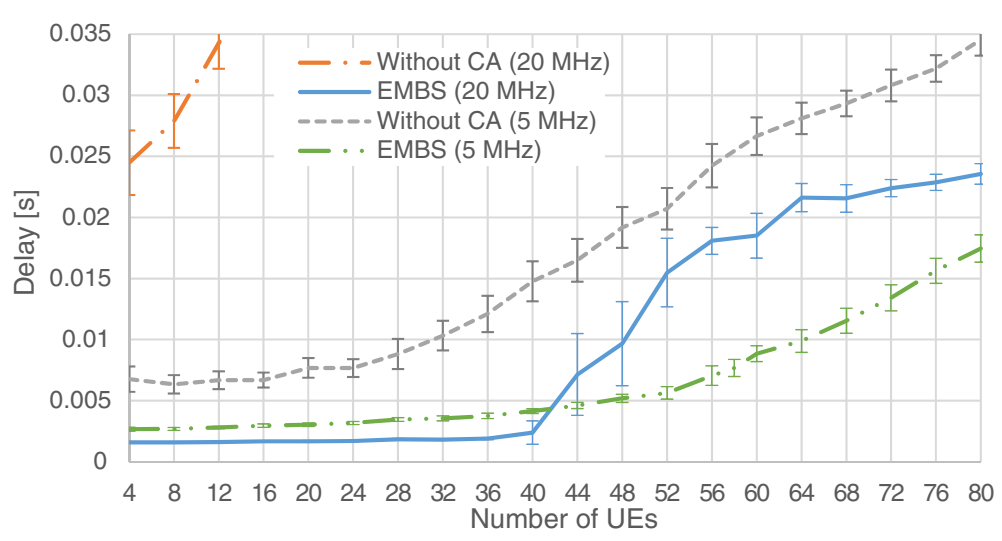

Figure 7 Comparison of the average delay in a cell as a function of the number of UEs for CA between scenarios with 5 and $20 \mathrm{MHz}$ CCs. 


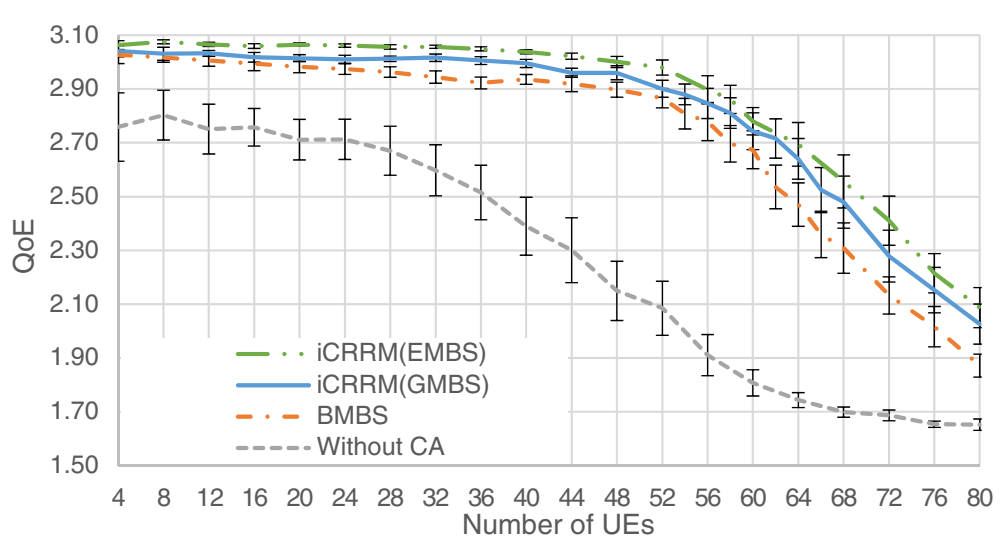

Figure 8 QoE as a function of the number of active UEs in the cell for $R=1,000 \mathrm{~m}$ (by applying CRRM or iCRRM, the QoE substantially increases, compared to the case without (A).

corresponding to a value of the QoE of circa 2.81 and 2.86, respectively.

In terms of QoE, it is also worthwhile to compare the results between the former $5-\mathrm{MHz}$ bandwidth scenario and the $20-\mathrm{MHz}$ bandwidth one (video with $b_{\text {rate }}=$ $3.1 \mathrm{Mbps}$ ). Figure 9 shows the predicted average cell QoE as a function of the number of active UEs, in cells with cell radius $R=1,000 \mathrm{~m}$.

It is observable that for the $20-\mathrm{MHz}$ bandwidth without $\mathrm{CA}$, the behaviour of $\mathrm{QoE}$ is not as regular as it was for the $5-\mathrm{MHz}$ bandwidth. However, when EMBS is employed, the QoE curve recovers its regular behaviour. It is also clear that the values of QoE raise from around 2.86 up to circa 3.96 (the value of QoE that corresponds to PLR $=1 \%$ for the 5-MHz and 20-MHz bandwidths, respectively).

\section{Goodput}

The variation of the supported average goodput with the number of UEs is shown in Figure 10 considering $R=$ $1,000 \mathrm{~m}$ and CCs with bandwidth of $5 \mathrm{MHz}$. In this case, the performance gap between iCRRM, CRRM and BMBS is less apparent. With the exception of the case without $\mathrm{CA}$, all the remaining scenarios can support the cell traffic requirement for PLR $=1 \%$ up to approximately 54,58 and 58 UEs with CRRM (BMBS), iCRRM (GMBS) and iCRRM (EMBS), respectively. However, it is clear that as the number of UEs within the cell increases so does the iCRRM performance gain, in comparison with the results both widths CRRM and without CA results. In the context of the iCRRM, it has also been shown that above 58 UEs, the goodput obtained with the EMBS is higher than the one obtained with the GMBS.

Additionally, it is also important to consider the supported goodput within ITU-T G.1010 [27] and 3GPP TS 22.105 [28] performance target. In this context, considering the number of UE supported within the $1 \%$ PLR margin, i.e. 58 and 54 UEs, with iCRRM and CRRM (BMBS), respectively, the supported goodput improvement between both RRM is evident, as shown in Figure 11. With iCRRM, an average of 7,480 and

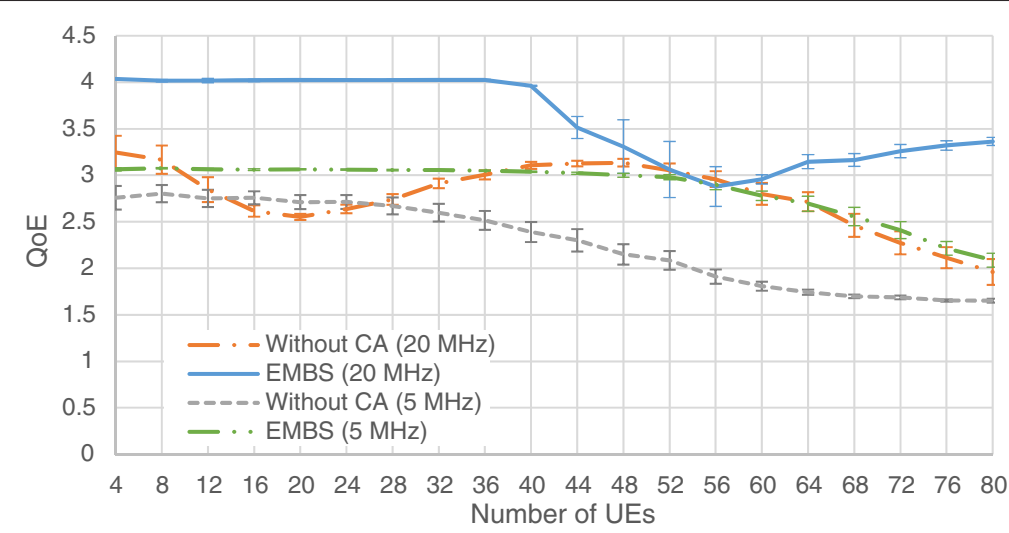

Figure 9 Comparison of the predicted average cell QoE as a function of the number of UEs between scenarios with 5 and $20 \mathrm{MHz} C \mathrm{Cs}$ for $R=1,000 \mathrm{~m}$ (the use of the 20-MHz bandwidth allows for considerable increase in QoE). 


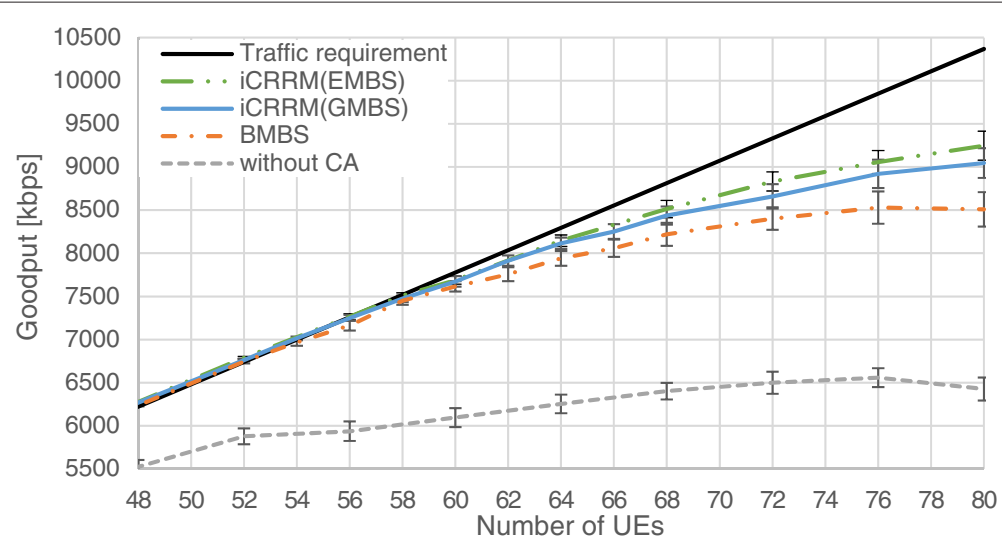

Figure 10 Average cell supported goodput as function of the number of UEs for $R=1,000 \mathrm{~m}$ (EMBS over performs GMBS and BMBS).

7,400 kbps are supported, with the EMBS and GMBS, respectively, whereas only $6,900 \mathrm{kbps}$ is supported with CRRM (BMBS). The case without CA is not considered, since the lowest obtained PLR is approximately $2 \%$.

Similarly to the above ITU-T and 3GPP target performance considerations, one should bear in mind the ITU-T ACR scale [34] whose lower QoE value is 1 and highest is 5 . When a bandwidth of $5 \mathrm{MHz}$ is considered, as the delay threshold is not reached, the parameter that limits the definition of the QoE threshold is the PLR (which should be less than 1\%). A value of 2.86 was identified for the EMBS (58 UEs), while CRRM (BMBS) and GMBS correspond to a value of 2.81 for QoE (54 and 58 UEs, respectively). A value equal to 2.86 , achieved for 58,54 and 52 users (for EMBS, GMBS and BMBS, respectively), is henceforward considered as a threshold below which the QoE is not acceptable. In this context, considering the results from Figure 8, without CA, the average cell QoE is never considered sufficient. With CA, the 2.86 threshold is no longer achieved above the same number of UEs as for the previous PLR analysis. Finally, the number of UEs supported by the cell below this QoE threshold can also be reflected in terms of average supported cell goodput, as shown in Figure 12. Given these considerations, the average supported goodput is approximately 7,480, 7,010, and 6,740 kbps with iCRRM (EMBS), iCRRM (GMBS) and CRRM (BMBS), respectively.

The analysis of the behaviour from the goodput while considering the $20-\mathrm{MHz}$ bandwidth leads us to the curves of the cell average goodput as a function of the number of UEs from Figure 13, where $R=1,000 \mathrm{~m}$ is assumed. With EMBS and 5-MHz bandwidth, a maximum value of $9.2 \mathrm{Mbps}$ is obtained for the average cell goodput (for 80 UEs, here PLR is circa 7\%), whereas for the $20-\mathrm{MHz}$ bandwidth the goodput is linearly increasing until it reaches a value of 71.53 to $75.30 \mathrm{Mbps}$ (for 40 to 44 UEs); then it takes slightly lower values. Without CA, values of only

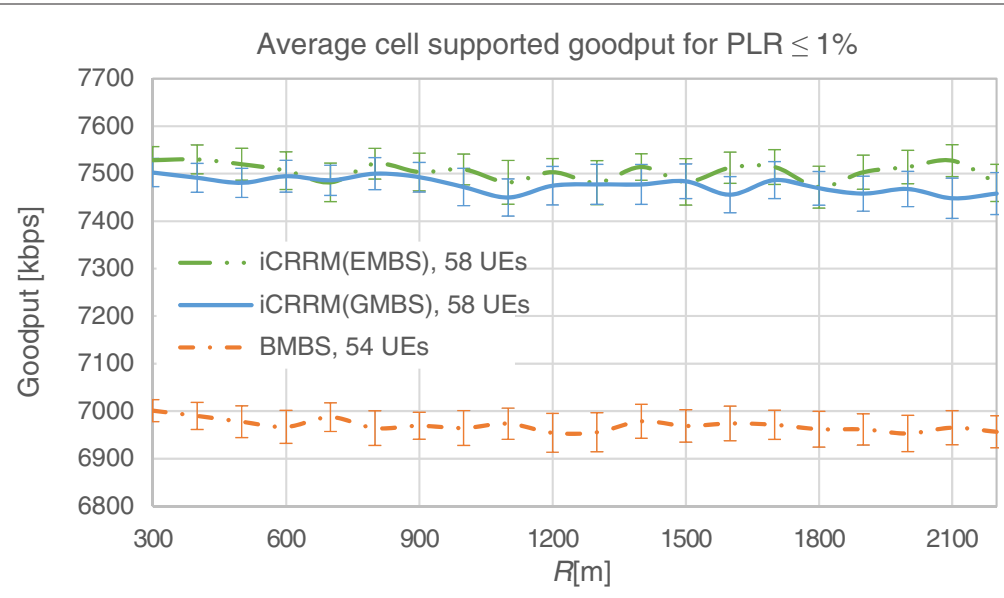

Figure 11 Average supported cell goodput as a function of the cell radius with $P L R \leq 1 \%$. 


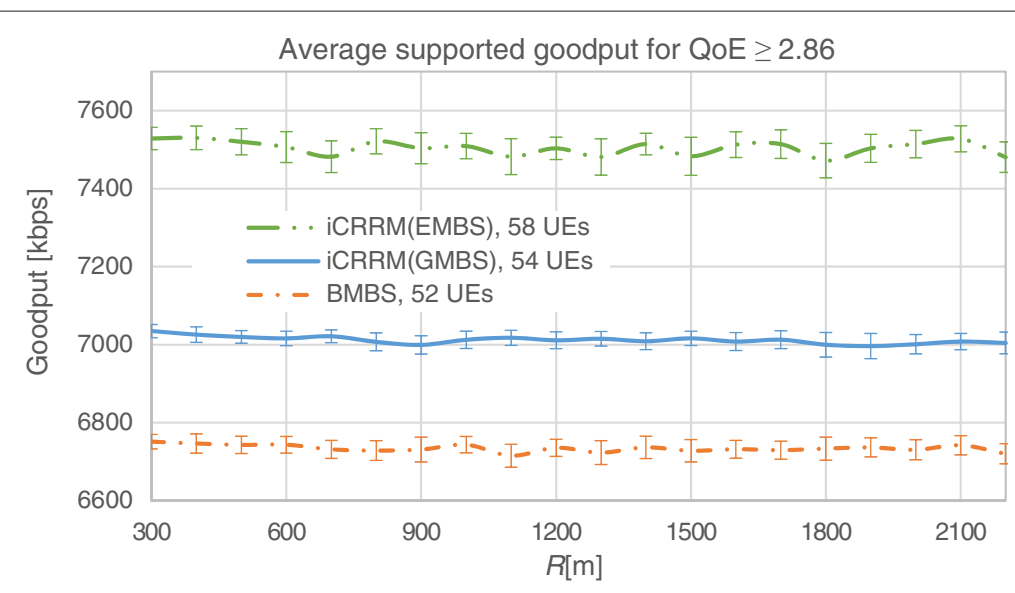

Figure 12 Average supported cell goodput as a function of the cell radius for QoE $\geq 2.86$ for different cell radii.

6.5 and 41.2 Mbps are achieved (for 76 and 80 UEs, respectively).

It is worthwhile to analyse the value for the goodput which corresponds to number of users supported under the $1 \%$ PLR performance target (since the 150-ms threshold has not been reached). In this context, with EMBS, the corresponding values for the average supported cell goodput are approximately 7.48 and $71.53 \mathrm{Mbps}$ (9.56 times increase), for the 5 and $20 \mathrm{MHz}$ (58 and $40 \mathrm{UEs}$ ), respectively. Results without CA are not considered since the PLR performance target is always exceeded.

Figure 14 shows the average supported cell goodput as a function of the cell radius, $R$, for the number of users supported under the 1\% PLR threshold. In this case, the formulation to compute the transmitter power required to guarantee a similar average SINR for different values of the cell radius is the one from [11]. The transmitter power has been normalized so that comparable results between $\mathrm{CCs}$ are assured and eNBs from lower cell radius have reduced energy consumption.
The average cell spectral efficiency has been computed as the ratio between the goodput and the CCs bandwidth, i.e. 5 and $20 \mathrm{MHz}$ (for 128-kbps and 3.1-Mbps video clips). Figure 15 shows the average cell spectral efficiency and corresponding percentage of gain (between EMBS and without CA). Considering the number of UEs, supported under the PLR threshold, with EMBS, the value for the spectral efficiency is $1.788 \mathrm{~b} / \mathrm{Hz} /$ cell (for $40 \mathrm{UEs}$ ) and $0.889 \mathrm{~b} / \mathrm{Hz} /$ cell without $\mathrm{CA}$, in the $20-\mathrm{MHz}$ case. In turn, for bandwidth of $5 \mathrm{MHz}$, the spectral efficiency is only $0.75 \mathrm{~b} / \mathrm{Hz} /$ cell (for $58 \mathrm{UEs}$ ) and $0.62 \mathrm{~b} / \mathrm{Hz} /$ cell without CA. Compared to the case without $\mathrm{CA}$, the corresponding percentage of gain is $101.2 \%$ (for 40 UEs) and circa 24\% (for 58 UEs), respectively.

\section{Cost/revenue analysis}

From the economic point of view, the different entities from cellular systems, such as subscribers, network operators, service providers, regulators and equipment vendors, should be taken into account [35]. In this research,

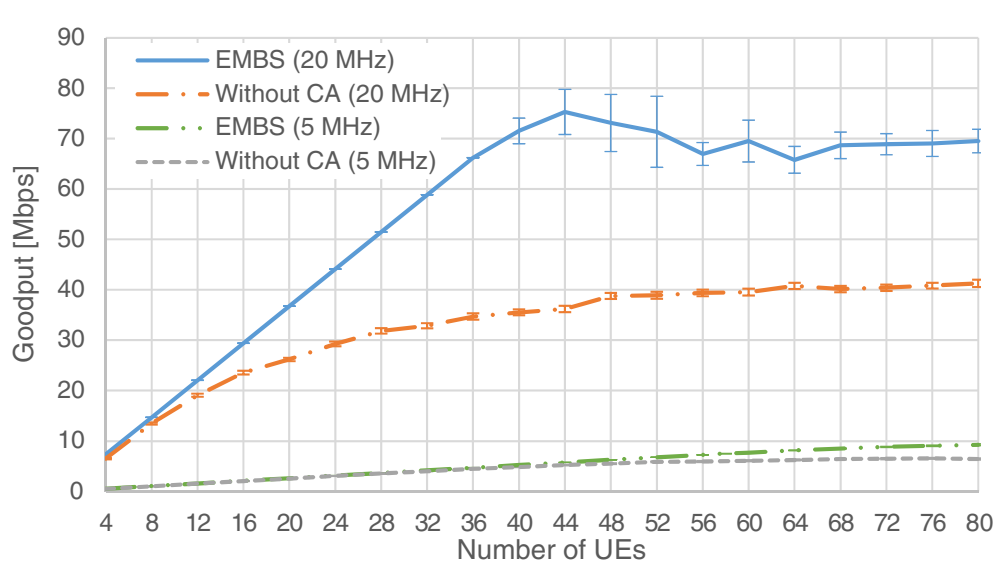

Figure 13 Average cell supported goodput as a function of the number of UEs. 




Figure 14 Average cell supported goodput as a function of the cell radius with $P L R \leq 1 \%$.

one considers operator's/service provider's point of view, whose main goal is to obtain the maximum profit from his business, i.e. to increase revenue, decreasing costs as much as possible. In this paper, costs and revenues are analysed on an annual basis, although a project duration of 5 years is assumed. Moreover, the analysis is performed under the assumption of a null discount rate. Nevertheless, this work does not intend to perform a complete economic study but aims at simply presenting initial contributions to understand the basic limits of using CA and facilitates cellular planning optimisation. Appropriate enhancements would be required, in order to perform a complete economic analysis based on discounted cash flows (e.g. to compute the net present value) while including the contribution from other relevant business aspects. From a cellular planning and radio resource management perspective, the objective of the operator is to determine an optimal operating point that maximizes the expected revenue. Examples of major decisions affecting this include the type of technology to be used, the size of the cell and the number of radio resources in use in each cell. It is therefore extremely important to identify the main components of the system's costs and revenues, in particular those that allow a direct relationship to either the maximum cell coverage distance or the reuse pattern, $K$. We consider the cost per unit area of a $2 \mathrm{D}$ system incurred during the system lifetime. The system is considered to have a transmission structure formed by a set of frequency carriers or channels (or the corresponding LTE sub-channels), each supporting a TDM frame structure. Each base station comprises a number of transceivers equal to the number of carriers assigned to the enode B (or to the enode B sector), which is assumed to be one in this study. This means that it is assumed as a simplification that one carrier will be sufficient per cell/sector. System cost has two major parts: (a) capital costs (normal backhaul, cell site planning and installation) and (b) operating expenses (operation, administration and



Figure 15 Average spectral efficiency and corresponding percentage of gain for bandwidths of 5 and $20 \mathrm{MHz}$ with PLR $\leq 1 \%$ (four times increase in spectrum resources corresponds to 2.39 times increase in spectral efficiency, from 0.75 to 1.788 b/Hz/cell). 
maintenance) $[36,37]$. The capital cost is taken to consist of:

- A fixed part (e.g. licensing and spectrum auctions or fees);

- A part proportional to the number of eNBs per kilometre or square kilometre (e.g. the installation costs of BSs including the cost of obtaining cell sites, the normal backhaul and the cost of hardware and core equipment common to all);

- A part proportional to the total number of transceivers per kilometre or square kilometre (e.g. the cost of the transceivers).

It is assumed that the cost of the connection between eNBs and the MME/GW, that is, the fixed part of the network (e.g. the cost of laying fibre), is not a fixed cost. Instead, we consider this to be proportional to the number of BSs/eNBs, which can be true if, for example, the mobile operator's service is contracted from a fixed network operator.

The operating cost during a system's lifetime is taken to contain:

- A part proportional to the number of eNBs per kilometre or square kilometre;

- A part proportional to the number of transceivers per kilometre or square kilometre.

These costs will be incurred on an annual basis.

In this context, the cost per unit area is given by [35]:

$$
C_{\left[€ / \mathrm{km}^{2}\right]}=C_{f i\left[€ / \mathrm{km}^{2}\right]}+C_{b[€ / \mathrm{cell}]} N_{\left[\mathrm{cell} / \mathrm{km}^{2}\right]}
$$

where $C_{f i}$ is the fixed term of the costs (e.g. licensing and spectrum auctions or fees), and $C_{b}$ is the cost per $\mathrm{BS} /$ enode $\mathrm{B}$. The number of hexagonal cells per unit area is given by:

$$
N_{\text {[cell } \left./ \mathrm{km}^{2}\right]}=\frac{2}{3 \sqrt{3} R^{2}}
$$

where $R$ represents the cell radius. Then, the total cost of the BS site, considering every element in the infrastructure, is given by:

$$
C_{b[€ / \text { cell }]}=\frac{C_{\mathrm{BS}}+C_{\mathrm{bh}}+C_{\mathrm{inst}}}{N_{\text {year }}}+C_{\mathrm{M} \& \mathrm{O}}
$$

where $N_{\text {year }}=5$ is the project's lifetime, $C_{\mathrm{BS}}$ is the cost of the $\mathrm{BS} / \mathrm{eNB}, C_{\mathrm{bh}}$ is the backhaul cost, $C_{\text {inst }}$ is the installation cost of the $\mathrm{BS}$ and $C_{\mathrm{M} \& \mathrm{O}}$ is the operation and maintenance cost.

The revenue in cell per year, $\left(R_{v}\right)_{\text {cell }}$, can be obtained as a function of the supported throughput per eNB,
$R_{b-\sup [\mathrm{kbps}]}$, and the revenue of a channel with a data rate $R_{b[\mathrm{kbps}]}, R_{R_{b}}[€ /$ MByte], by:

$\left(R_{v}\right)_{\text {cell }}=\frac{N_{\text {hex }\left[\mathrm{km}^{2}\right]} \cdot R_{(b-\text { sup })_{\text {equiv }}[\mathrm{kbps}]} \cdot T_{\mathrm{bh}} \cdot R_{R_{b}[€ / \mathrm{min}]}}{R_{b-\mathrm{ch}[\mathrm{kbps}]}}$

where $N_{\text {hex }}$ is the number of hexagonal areas, $T_{\mathrm{bh}}$ is the equivalent duration of busy hours per day, and $R_{b-c h}$ is the bit rate of the basic "channel".

The revenue per unit area per year, $R_{v\left[€ / \mathrm{km}^{2}\right]}$, is obtained by multiplying the revenue per cell by the number of cells per unit area:

$R_{\nu}\left[€ / \mathrm{km}^{2}\right]=N_{\text {hex }\left[\mathrm{km}^{2}\right]} \cdot\left(R_{\nu}\right)_{\text {cell }}=N_{\text {hex }\left[\mathrm{km}^{2}\right]} \cdot \frac{R_{b-\mathrm{sup}[\mathrm{kbps}]} \cdot T_{\mathrm{bh}} \cdot R_{R_{b}[€ / \mathrm{min}]}}{R_{b-\mathrm{ch}[\mathrm{kbps}]}}$

The (absolute) profit is given by:

$$
P_{\left[€ / \mathrm{km}^{2}\right]}=R_{v}-C
$$

from which the profit in percentage terms (compared to the costs) is given by:

$$
P_{[\%]}=\frac{R_{v}-C}{C} \times 100
$$

One considers a LTE system deployed in Portugal. The $\mathrm{BS} / \mathrm{eNB}$ cost $C_{\mathrm{BS}}$ is assumed to be the value referenced in [38], i.e. $C_{\mathrm{BS}}=10,000 €$, The $\mathrm{BS}$ installation cost, $C_{\text {inst }}=22,500 €$, is obtained by assuming $2,500 €$ for the radio installation plus $20,000 €$ for the infrastructure cost, e.g. site acquisition, site design and site construction. The backhaul cost is considered to be $C_{\mathrm{bh}}=5,000 €$. Finally, the operation and maintenance costs which include firstline maintenance, rental costs and preventive and corrective infra maintenance is $C_{\mathrm{M} \& \mathrm{O}}=1,500 €$ per year of operation. For a project duration of $N_{\text {year }}=5$, one obtains $C_{b}=9,000 €$ per BS/eNB.

Bearing in mind ANACOM's auction results [12], it is known that each $2 \times 5 \mathrm{MHz}$ of bandwidth was sold for $45,000,000 €$ and $3,000,000 €$ for the $800 \mathrm{MHz}$ and $2.6 \mathrm{GHz}$ CCs, respectively. Considering reuse pattern $K=$ 3 , the total license cost for the $800 \mathrm{MHz}$ and $2.6 \mathrm{GHz}$ are $3 \times 4,000,000 €=135,000,000 €$ and $3 \times 3,000,000 €=$ $9,000,000 €$, respectively (5-MHz bandwidth). Assuming these values and considering a total area of $91,391.5 \mathrm{~km}^{2}$ as the area of Portugal, the fixed cost per unit area is as follows:

$$
\begin{aligned}
C_{\mathrm{fi}} 800 \mathrm{MHz} & =\frac{135,000,000}{91,391.5 \times 5} \approx 295\left[€ / \mathrm{km}^{2}\right] \\
C_{\mathrm{fi}} 2.6 \mathrm{GHz} & =\frac{9,000,000}{91,391.5 \times 5} \approx 19.70\left[€ / \mathrm{km}^{2}\right]
\end{aligned}
$$

A summary of the cost assumptions is presented in Table 3, where we also show the fixed costs for the case of 20-MHz bandwidth, obtained by multiplying the former 
Table 3 Assumptions for costs

\begin{tabular}{lll}
\hline Costs & $\mathbf{5 ~ M H z}$ & $\mathbf{2 0 ~} \mathbf{~ H z}$ \\
\hline$C_{f i} 800 \mathrm{MHz}\left[€ / \mathrm{km}^{2}\right]$ & 295.00 & $1,180.00$ \\
$C_{\mathrm{fi} 2.6 \mathrm{GHz}\left[€ / \mathrm{km}^{2}\right]}$ & 19.70 & 78.80 \\
$C_{\mathrm{BS}[€]}$ & 10,000 & 11,000 \\
$C_{\text {inst }[€]}$ & 22,500 & 22,500 \\
$C_{\text {bh }[€]}$ & 5,000 & 5,500 \\
$C_{\mathrm{M \& O}[€]}$ & 1,500 & 1,500 \\
\hline
\end{tabular}

fixed costs by 4 . We assume that for the $20-\mathrm{MHz}$ bandwidth case, the cost of the BS and from the backhaul is $10 \%$ higher. The comparison between the use of $5-\mathrm{MHz}$ and 20-MHz bandwidths explores the impact of the much higher fixed cost in the economic feasibility of such $4 \mathrm{G}$ networks by trading off cost with statistical multiplexing gain from higher bandwidth availability and the price per megabyte of traffic.

Although nowadays the trend is to consider flat rate/fee for data and multimedia traffic revenues, in this work, one still considers the price per megabyte, $\mathrm{MB}$, of information, $R_{144[€ / M B y t e]}$ (N.B.: the price per minute approximately corresponds to the price of 1 MByte, as $144 \times$ $60=8,640 \mathrm{~kb} \approx 1$ MByte). In this context, for the $5-\mathrm{MHz}$ bandwidth case, two values for the price of the basic channel are considered, $R_{144[€ / \text { MByte] }}=0.005$ and $R_{144[€ / \text { MByte] }}=0.01$. For the $20-\mathrm{MHz}$ bandwidth case, the prices are one fifth of the above ones, i.e. $R_{144[€ / \text { MByte] }}=0.001$ and 0.002 , respectively. Assuming the above assumptions for the costs and channel price, as well as the simulation average supported goodput results, $R_{b-\text { sup [kbps] }}$, considering an equivalent duration of busy hours per day, $T_{\mathrm{bh}}$, equal to 6 busy hours per day, as well as 240 busy days per year, one computes the total cost, $C_{\text {total }}$ and the total revenue per unit area per year, $R_{v}$ total $\left(R_{v\left[€ / \mathrm{km}^{2}\right]}\right)$. The latter considers the simulation results of the supported goodput $R_{b-\text { sup [kbps] }}$, obtained for saturation conditions, i.e. maximum PLR less or equal to $1 \%$ for the considered range of coverage distances, while assuming that this is the traffic during the busiest hours. Figure 16 shows that, considering iCRRM (EMBS) for the 5-MHz bandwidth case, with 58 users, $C_{\text {total }}$ and $R_{v_{\text {total }}}$ decrease as the cell radius increases, the revenues are higher than the total cost and higher revenues are obtained with higher prices for the traffic. A logarithmic scale is considered for the $y y$ axis in order to enable the comparison between the two different curves for the cost. For the $5-\mathrm{MHz}$ bandwidth case, the cost is considerably much lower than for the $20-\mathrm{MHz}$ bandwidth case. For the $5-\mathrm{MHz}$ case and price $R_{144[€ / \mathrm{MByte}]}=$ 0.005 , the revenue is approximately equal to (just slightly higher than) the revenue for a price of $0.001 € /$ MByte and $20-\mathrm{MHz}$ bandwidth. The 9.56 times increase in the suported goodput (from 7.48 to $71.53 \mathrm{Mbps}$ ) compensates the five times reduction in price. For the 20$\mathrm{MHz}$ bandwidth case, as expected, for twice of the price, i.e. $0.002 € /$ MByte, the revenue is considerably higher.

To analyse the profit in percentage for PLR $\leq 1 c$, for the $5-\mathrm{MHz}$ bandwidth case, one considers the average supported goodput while considering iCRRM (EMBS), iCRRM (GMBS) and CRRM (BMBS). It is worthwhile to note that, as the 1\% PLR performance threshold is not achieved without CA, this scenario will not be considered. Figures 17 and 18 show the profit in percentage for PLR $\leq 1 \%$. While for the $5-\mathrm{MHz}$ bandwidth, EMBS, GMBS and BMBS are considered and prices are $R_{144[€ / \text { MByte }]}=0.005$ and $R_{144[€ / \mathrm{MByte}]}=0.01$, respectively, for the $20-\mathrm{MHz}$ bandwith case, only EMBS is addressed and the respective prices are $R_{144[€ / \text { MByte] }}=0.001$ and 0.002 .

One could also investigate the $R_{b-\text { sup [kbps] }}$ reached with $\mathrm{QoE} \geq 2.86$. However, we skip this analysis as the values from the goodput for PLR $\leq 1 \%$ correspond to $\mathrm{QoE} \geq$



Figure 16 Total cost and revenue for different cell radii, for prices $R_{144[€ / \mathrm{MByte}]}=0.005$ and 0.01 (5-MHz bandwidth), and $R_{144[€ / \mathrm{MBy} t e]}=0.001$ and 0.002 (20-MHz bandwidth). 


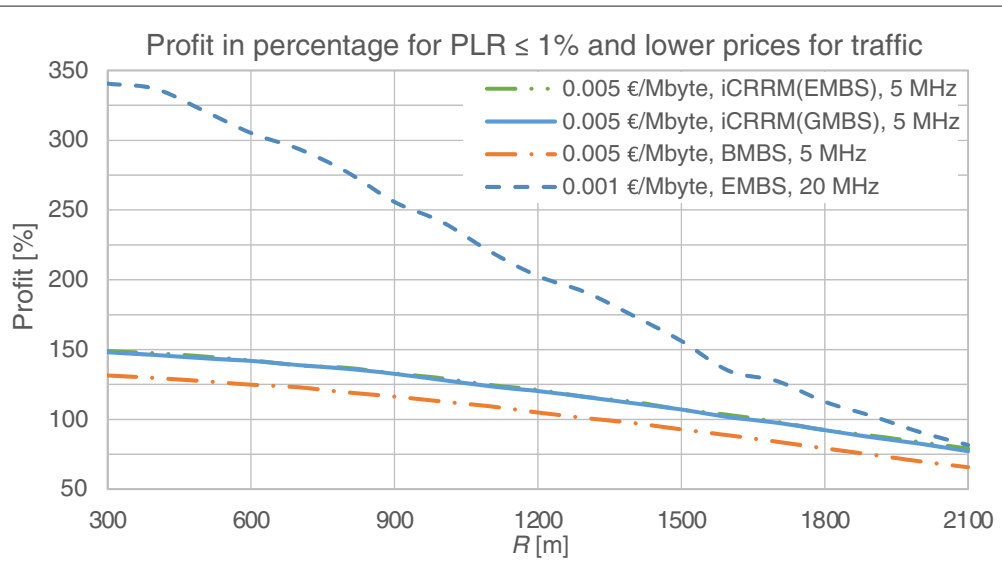

Figure 17 Profit in percentage terms as a function of the cell radius and PLR $\leq 1 \%$, for $R_{144[€ / M B y t e]}=0.005$ (5-MHz bandwidth) and $R_{144[€ / \mathrm{MByte}]}=0.001$ (20-MHz bandwidth).

2.86 for EMBS (58 supported users), and only for GMBS and BMBS the values from the goodput (and PLR) are slightly lower for QoE $\geq 2.86$, as 54 and 52 users are supported, instead of 58 and 54, respectively.

Overall, it is evident that the profit increases as the price per $\mathrm{MB}$ increases. Besides, it decreases with the increase of the cell radius. In the $5-\mathrm{MHz}$ bandwidth case, considering PLR $\leq 1 \%$, the CRRM presents the lowest results for the profit, whereas iCRRM's EMBS and GMBS reach comparable profits as their respective goodputs are also similar. Employing iCRRM's EMBS and considering $R=1,000 \mathrm{~m}$ and PLR $\leq 1 \%$, profits of $130 \%$ or $360 \%$ are obtained with $R_{144[€ / M B y t e]}$ equal to 0.005 and 0.01 , respectively. With iCRRM's GMBS, profits of $129 \%$ or $357 \%$ are achieved considering $R_{144[€ / \mathrm{MByte}]}$ equal to 0.005 and 0.01 , respectively. Considering the basic CRRM, profits of $113 \%$ or $326 \%$ are obtained for PLR $\leq 1 \%$, with prices of 0.005 and $0.01 € /$ MByte, respectively.
In Figures 17 and 18, for the 20-MHz case, the profit decreases at a considerably higher rate (compared to $5-\mathrm{MHz}$ bandwidth), which is due to the lower rate of decrease from the curve of the costs, as shown in Figure 16. The comparison of the profit in percentage between these two values for the bandwidth from Figure 19 shows that with a price of $0.001 € /$ MByte, the curve for the profit considering the $20-\mathrm{MHz}$ bandwidth is similar to the one for price of $0.01 € /$ MByte considering $5-\mathrm{MHz}$ bandwidth, mainly for the smallest cell sizes (few hundred meters). Then, it starts to decrease faster. For example, for $R=1,000 \mathrm{~m}$, it is clearly shown that with a price of $1 / 5,0.001$ or $0.002 € /$ MByte for the $20-\mathrm{MHz}$ case (compared to 0.005 or $0.01 € / M B y t e$ for the $5-\mathrm{MHz}$ bandwidth), values for the profit are $241 \%$ or $583 \%$ (against $130 \%$ or $360 \%$ for the $5-\mathrm{MHz}$ bandwidth), i.e. respectively $241 / 130=1.85$ or $583 / 360=1.62$ times higher than the ones from the $5-\mathrm{MHz}$ bandwidth.

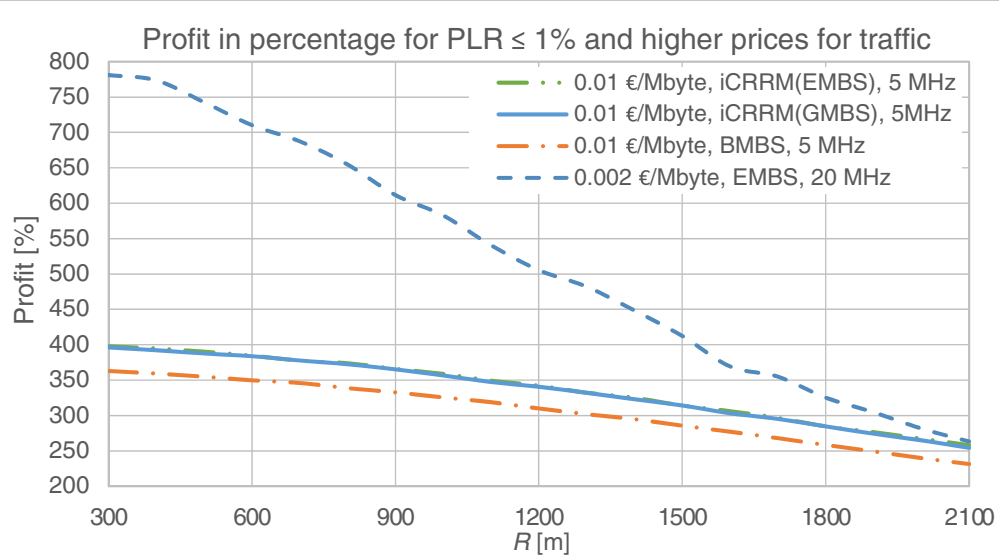

Figure 18 Profit in percentage as a function of the cell radius and PLR $\leq 1 \%$, for $R_{144[€ / \mathrm{MByte}]}=0.01$ (5-MHz bandwidth) and $R_{144[€ / \mathrm{MByte}]}=0.002$ (20-MHz bandwidth). 


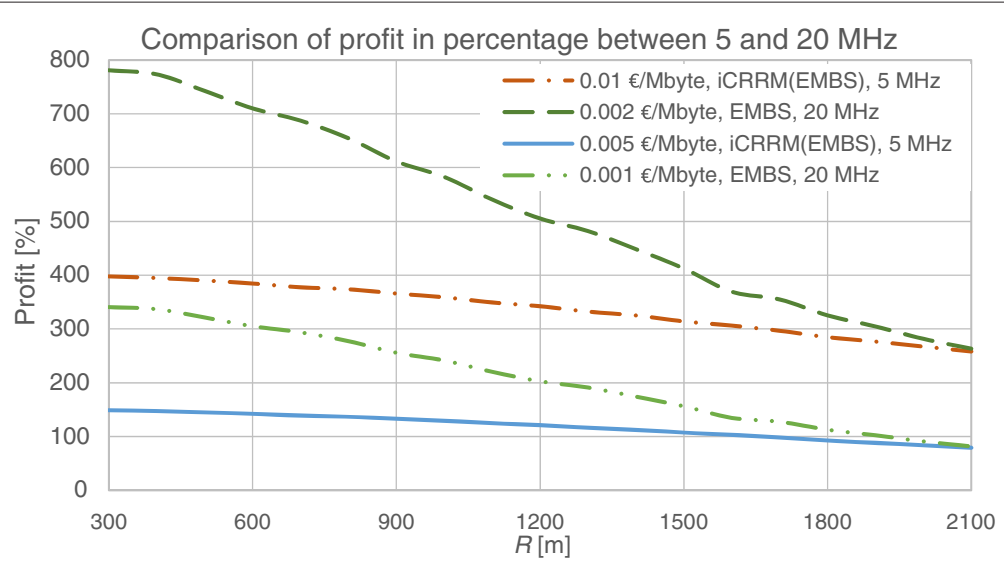

Figure 19 Comparison of profit in percentage terms as a function of the cell radius between 5 - and 20-MHz bandwidths, with the price per megabyte as a parameter.

While the cell radius increases, as the costs are higher for the 20-MHz bandwidth (mainly owing to the four times higher cost of the license, $C_{\mathrm{fi}}$, grey curve in Figure 16), the profit in percentage decreases faster, which motivates the use of small(er) cells.

\section{Conclusions}

This work proposes an adaptation and update of the integrated CRRM entity proposed in [11] applied to LTE-A. The iCRRM entity implements inter-band carrier aggregation (CA) by performing scheduling between two component carriers $(\mathrm{CCs})$, i.e. band $7(2.6 \mathrm{GHz})$ and band $20(800 \mathrm{MHz})$, with the aim of increasing users' service quality requirements and improve spectral usage. Besides, iCRRM may operate with one of two multi-band schedulers. The general multi-band schedulers (GMBS) operate in parallel with a classic downlink packet schedulers, i.e. iCRRM assigns users to one CC and the scheduling of resource blocks (RBs) of each CC (to the allocated users) is performed by the M-LWDF packet scheduler. In turn, the proposed enhanced multi-band scheduler (EMBS) operates on its own, is less complex and allocates RBs from both CCs. Moreover, with the GMBS UEs can only be allocated to one $\mathrm{CC}$ at a time whereas with EMBS UEs can be allocated to both CCs. Additionally, considering available bandwidths auctioned by the Portuguese communication regulator, ANACOM, in 2011, the research on CA from this work was addressed considering first $5-\mathrm{MHz}$ bandwidth CCs and then $20-\mathrm{MHz}$ bandwidth CCs, in scenarios that resemble the South Korea ones. Furthermore, bearing in mind CISCO's mobile traffic forecast, e.g. video traffic embodied $53 \%$ of all traffic in 2013 and will reach $69 \%$ by 2018 , one addressed this work considering that each user generates one video traffic flow. In the simulations, each flow is characterized as one trace based H.264 128-kbps video bit rate flow. Additionally, through extensive simulations, it has been found that the modified largest weighted delay first (M-LWDF) scheduler provided the best results for this type of traffic. Hence, it was selected to operate in conjunction with iCRRM's GMBS.

To analyse the iCRRM performance with several cell radii with comparable conditions and to reduce energy consumption by reducing the transmitter power for low cell radii, the average cell interference-plus-noise ratio (SINR) must be kept constant. In this context, the formulation proposed in [11] and [20] to compute the transmitter power needed to cover cells of different sizes while maintaining the average cell SINR constant and near the maximum, considering frequency reuse pattern three, was considered. The normalized transmitter power required to maintain such average cell SINR was computed and used within the simulation framework.

Extensive simulations were performed with LTE-Sim, and the performance analysis was performed addressing ITU-T G.1010 [27] and 3GPP TS 22.105 [28] performance targets and the corresponding supported goodputs. For the $5-\mathrm{MHz}$ bandwidth case, simulation results have shown that the $1 \%$ packet loss ratio (PLR) threshold is only exceeded above 58 and 54 UEs with iCRRM (EMBS and GMBS) and CRRM (BMBS), respectively. Without CA, the minimum obtained PLR is approximately $2 \%$. In this condition, the average supported cell goodput is approximately 7,480 and 7,400 kbps with iCRRM EMBS and GMBS, respectively, and 6,900 kbps with CRRM (BMBS). The ITU-T G.1010 [27] and 3GPP TS 22.105 [28] 150ms preferred average cell delay performance target has not been reached in the performed simulations. For 20$\mathrm{MHz}$ bandwidth, only iCRRM (EMBS) was considered. With 40 users, the 1\% PLR threshold is not exceeded and the supported goodput is 71.53 Mbps. Overall, a gain of circa 9.56 times occurs in the average supported cell goodput, from approximately 7.48 to $71.53 \mathrm{Mbps}$, for the 5- and 20-MHz bandwidths (58 and 40 UEs), respectively. 
Considering the unified model for the mapping of quality of service (QoS) parameters into quality of experience (QoE) from [33], the perceived impact of CA has been estimated. It has been found that, for the $5-\mathrm{MHz}$ bandwidth, a minimum QoE of 2.86 can only be supported up to 58, 54 and 52 UEs with EMBS, GMBS and iCRRM's GMBS, respectively. Moreover, the average cell goodput equivalent to the number of UEs supported within this QoE threshold is approximately 7,480, 7,010, and $6,740 \mathrm{kbps}$ with the EMBS, GMBS and BMBS, respectively. For the $20-\mathrm{MHz}$ bandwith case, the values of the QoE considerably increase to values of circa 3.96 (corresponding to PLR $=1 \%$ ).

The cost/revenue analysis was performed considering the formulation from [35] and assuming values for the goodput under the PLR $\leq 1 \%$ performance target. It was observed that prices can be much lower if a bandwidth of $20 \mathrm{MHz}$ is available, instead of $5 \mathrm{MHz}$. For the $5-\mathrm{MHz}$ bandwidth, two values of the price per MB have been considered, $R_{144[€ / \text { MByte] }}$ equal to 0.005 or 0.01 , whereas for the $20-\mathrm{MHz}$ bandwidth prices are $1 / 5$ of the former ones, i.e. 0.001 or $0.002 € /$ MByte. It has been found that without CA, the profit cannot be evaluated under PLR $\leq 1 \%$ since this constraint is not satisfied. It is evident that the profit increases as the price per MB increases. Besides, it decreases with the increase of the cell radius. Besides, for the $5-\mathrm{MHz}$ bandwidth, for PLR $\leq 1 \%$ iCRRM's EMBS and GMBS, the values of the profit in percentage are the highest and yet similar, since the number of supported UEs is alike. For $R=1,000 \mathrm{~m}$ and PLR $\leq 1 \%$, profits of $130 \%$ or $360 \%, 129 \%$ or $357 \%$ and $113 \%$ or $326 \%$ were obtained for the EMBS, GMBS and BMBS, for $R_{144[€ / M B y t e]}$ equal to 0.005 and 0.01 , respectively.

From the analysis of the economic trade-off, for the 20$\mathrm{MHz}$ case, the profit decreases at a considerably higher rate (compared to $5-\mathrm{MHz}$ bandwidth), which is due to the lower rate of decrease from the curve of the costs, implying that for PLR $\leq 1 \%$ and a price of $0.001 € /$ MByte, the curve for the profit in percentage considering the $20-\mathrm{MHz}$ bandwidth is similar to the one for price of $0.01 € /$ MByte considering $5-\mathrm{MHz}$ bandwidth, mainly for the shortest cell sizes. Then, the profit in percentage terms starts to decrease faster. For example, for $R=1,000 \mathrm{~m}$, one can conclude that with a price of $1 / 5$, i.e. 0.001 or $0.002 € /$ MByte, for the $20-\mathrm{MHz}$ case (compared to 0.005 or $0.01 € /$ MByte for the $5-\mathrm{MHz}$ bandwidth), values for the profit are $241 \%$ or $583 \%$ (against $130 \%$ or $360 \%$ for the $5-\mathrm{MHz}$ bandwidth), i.e. respectively $241 / 130=1.85$ or $583 / 360=1.62$ times higher than the ones from the $5-\mathrm{MHz}$ bandwidth.

While the cell radius increases, as the costs are higher for the $20-\mathrm{MHz}$ bandwidth (mainly owing to the four times higher cost of the license, $C_{\mathrm{fi}}$, grey curve in Figure 16), the profit in percentage decreases faster, which motivates the use of small(er) cells. Moreover, the global implementation of CA should reduce the manufacturing and implementation costs of CA-enabled equipment, hence future work will assess cost/revenues analysis with up to date assumptions on prices. Besides, as the flat rate pricing model is being employed worldwide, upcoming work will also address the impact of $\mathrm{CA}$ in the cost/revenue trade-off under this business model.

\section{Competing interests}

The authors declare that they have no competing interests.

\section{Acknowledgements}

This work has been partially supported and funded by the FCT project PEst-OE/EEI/LA0008/2013, UID/EEA/50008/2013, OPPORTUNISTIC-CR, LTE-Advanced Enhancements using Femtocells, CREaTION, COST ICO905 "TERRA", COST IC 1004, and by the Marie Curie Reintegration Grant PLANOPTI (FP7-PEOPLE-2009-RG), EFATraS, E-COOP, HANDCAD and ORCIP. The authors also acknowledge Doctor Giuseppe Piro for his precious guidance in the implementation of spectrum aggregation functionalities in LTE-Sim.

Received: 9 August 2014 Accepted: 24 April 2015

Published online: 08 July 2015

\section{References}

1. H Lee, S Vahid, K Moessner, A survey of radio resource management for spectrum aggregation in LTE-Advanced. Communications Surveys Tutorials, IEEE. 16(2), 745-760 (2014). doi:10.1109/SURV.2013.101813.00275

2. 3GPP, Technical specifications and technical reports for a UTRAN based 3GPP system (Release 10). TS 22.101, 3rd Generation Partnership Project (3GPP) (September 2012). http://www.3gpp.org/ftp/Specs/html-info/ 22101.htm

3. X Lin, JG Andrews, A Ghosh, Modeling, analysis and design for carrier aggregation in heterogeneous cellular networks. Communications, IEEE Transactions on. 61(9), 4002-4015 (2013). doi:10.1109/TCOMM.2013.071813. 120880

4. 3GPP, Evolved Universal Terrestrial Radio Access (E-UTRA) and Evolved Universal Terrestrial Radio Access (E-UTRAN); Overall description; Stage 2. TS 36.300, 3rd Generation Partnership Project (3GPP) (September 2008). http://www.3gpp.org/ftp/Specs/html-info/36300.htm

5. F Liu, K Zheng, W Xiang, H Zhao, Design and performance analysis of an energy-efficient uplink carrier aggregation scheme. Selected Areas in Communications, IEEE Journal on. 32(2), 197-207 (2014). doi:10.1109/JSAC.2014.141202

6. Carrier Aggregation Explained. http://www.3gpp.org/technologies/ keywords-acronyms/101-carrier-aggregation-explained

7. Y Wang, KI Pedersen, PE Mogensen, TB Sorensen. Personal, Indoor and Mobile Radio Communications, 2009 IEEE 20th International Symposium On, (2009), pp. 370-374. doi:10.1109/PIMRC.2009.5450150

8. Y Y Wang, KI Pedersen, TB Sorensen, PE Mogensen, Carrier load balancing and packet scheduling for multi-carrier systems. Wireless Communications, IEEE Transactions on. 9(5), 1780-1789 (2010). doi:10.1109/TWC.2010.05.091310

9. Y Wang, KI Pedersen, TB Sorensen, PE Mogensen, in Vehicular Technology Conference (VTC Spring), 2011 IEEE 73rd. Utility maximization in LTE-Advanced systems with carrier aggregation, (2011), pp. 1-5. doi:10.1109/NETECS.2011.5956494

10. G Galaviz, D Covarrubias, A Andrade, S Villarreal, A resource block organization strategy for scheduling in carrier aggregated systems. EURASIP Journal on Wireless Communications and Networking. 2012(1) 107 (2012). doi:10.1186/1687-1499-2012-107

11. O Cabral, F Meucci, A Mihovska, FJ Velez, NR Prasad, R Prasad, Integrated common radio resource management with spectrum aggregation over non-contiguous frequency bands. Wireless Personal Communications. 59(3), 499-523 (2011). doi:10.1007/s11277-011-0242-6

12. Anacom, Auction regulation for the allocation of rights of use of frequencies in the $450 \mathrm{MHz}, 800 \mathrm{MHz}, 900 \mathrm{MHz}, 1800 \mathrm{MHz}, 2.1 \mathrm{GHz}$ and 2.6 GHz bands. Regulation, Anacom (October 2011). www.anacom.pt/ streaming/english_version_Auction_Regulation.pdf?contentld= 1101807\&field=ATTACHED_FILE 
13. SK, LG Eye 4 Times Faster LTE Service. http://www.koreaherald.com/view. php?ud=20140120000817

14. EE Debuts 300 Mbps LTE-A Network Using Huawei's Carrier Aggregation Router. http://www.mobileeurope.co.uk/press-wire/ee-launches300mbps-lte-a-network-using-huawei-s-carrier-aggregation-router

15. http://www.cellular-news.com/story/64585.php

16. SingTel Mobile Launches LTE-A 300Mbps Service. https://www. telegeography.com/products/commsupdate/articles/2014/06/02/ singtel-mobile-launches-lte-a-300mbps-service/

17. TD Boosts LTE Speeds to 300Mbps in Select Areas. https://www. telegeography.com/products/commsupdate/articles/2014/11/18/tdboosts-Ite-speeds-to-300mbps-in-select-areas/

18. Cisco visual networking index: global mobile data traffic forecast update. White Paper (2014)

19. H Wang, C Rosa, K Pedersen, in Communications (ICC), 2011 IEEE International Conference On. Uplink component carrier selection for Ite-advanced systems with carrier aggregation, (2011), pp. 1-5. doi:10.1109/icc.2011.5963279

20. J Acevedo, D Robalo, FJ Velez, Transmitted power formulation for the optimization of spectrum aggregation in Ite-a over $800 \mathrm{MHz}$ and $2 \mathrm{GHz}$ frequency bands. Special Issue of Wireless Personal Communications (accepted for publication with minor revisions) (2014)

21. GSMA report on Mobile Infrastructure Sharing. Report (2012). www.gsma. com/publicpolicy/wp-content/uploads/2012/09/Mobile-Infrastructuresharing.pdf

22. Radio Frequency (RF) System Scenarios. TR 25.942, 3rd Generation Partnership Project (3GPP) (June 2012), 3GPP. www.3gpp.org/ DynaReport/25942.htm

23. G Piro, LA Grieco, G Boggia, F Capozzi, P Camarda, Simulating LTE cellular systems: an open-source framework. Vehicular Technology, IEEE Transactions on. 60(2), 498-513 (2011). doi:10.1109/TVT.2010.2091660

24. F Capozzi, G Piro, LA Grieco, G Boggia, P Camarda, Downlink packet scheduling in LTE cellular networks: key design issues and a survey. Communications Surveys Tutorials, IEEE. 15(2), 678-700 (2013). doi:10.1109/SURV.2012.060912.00100

25. JK Karlof, Integer Programming: Theory and Practice. Operations Research Series. (Taylor \& Francis, London, 2005). http://books.google.pt/books?id= TO3XQO9XEjwC

26. TAli-Yahiya, Understanding LTE and Its Performance. SpringerLink: Bücher. (Springer, New York, 2011). http://books.google.pt/books?id= R_oLk8yFTkkC

27. ITU-T, End-user multimedia QoS categories. Recommendation G.1010, International Telecommunication Union, Geneva (November 2001)

28. 3GPP, Services and Service Capabilities. TS 22.105, 3rd Generation Partnership Project (3GPP) (December 2013). www.3gpp.org/ DynaReport/22105.htm

29. JA Hassan, SK Das, M Hassan, C Bisdikian, D Soldani, Improving quality of experience for network services [guest editorial]. Network, IEEE. 24(2), 4-6 (2010). doi:10.1109/MNET.2010.5430137

30. H Wang, C Rosa, K Pedersen, in Vehicular Technology Conference (VTC Fall), 2011 IEEE. Performance analysis of downlink inter-band carrier aggregation in Ite-advanced, (2011), pp. 1-5. doi:10.1109NETECF.2011.6092836

31. R Sivaraj, A Pande, K Zeng, K Govindan, P Mohapatra, in World of Wireless, Mobile and Multimedia Networks (WoWMoM), 2012 IEEE International Symposium on A. Edge-prioritized channel- and traffic-aware uplink carrier aggregation in LTE-advanced systems, (2012), pp. 1-9. doi:10.1109/WoWMoM.2012.6263691

32. P Brooks, B Hestnes, User measures of quality of experience: why being objective and quantitative is important. Network, IEEE. 24(2), 8-13 (2010). doi:10.1109/MNET.2010.5430138

33. FJV Daniel Robalo, in Vehicular Technology Conference (VTC Spring), 2014 IEEE 79th. A model for mapping between the quality of service and experience for wireless multimedia applications, (2014). doi:10.1109/MVT.2013.2260419

34. ITU-T, Methods for subjective determination of transmission quality. Recommendation P.800, International Telecommunication Union, Geneva (February 1996)

35. R Prasad, FJ Velez, WiMAX Networks: Techno-Economic Vision and Challenges, Dordrecht (2010). http://books.google.pt/books?id= hYZKAAAAQBAJ
36. DP Reed, The cost structure of personal communication services. Communications Magazine, IEEE. 31(4), 102-108 (1993). doi:10.1109/35.210403

37. J Sarnecki, C Vinodrai, A Javed, P O’Kelly, K Dick, Microcell design principles. Communications Magazine, IEEE. 31(4), 76-82 (1993). doi:10.1109/35.210399

38. A Ahmed, J Markendahl, C Cavdar, in Telecommunications (ICT), 201421 st International Conference On. Interplay between cost, capacity and power consumption in heterogeneous mobile networks, (2014), pp. 98-102. doi:10.1109/ICT.2014.6845088

\section{Submit your manuscript to a SpringerOpen ${ }^{\circ}$ journal and benefit from:}

- Convenient online submission

Rigorous peer review

- Immediate publication on acceptance

- Open access: articles freely available online

- High visibility within the field

- Retaining the copyright to your article

Submit your next manuscript at $>$ springeropen.com 